\title{
Sensemble: A Wireless, Compact, Multi-User Sensor System for Interactive Dance
}

\author{
Ryan Aylward \\ Responsive Environments \\ MIT Media Laboratory \\ 20 Ames St. \\ Cambridge, MA 01239 \\ 1-617-452-5647 \\ aylward@media.mit.edu
}

\author{
Joseph A. Paradiso \\ Responsive Environments \\ MIT Media Laboratory \\ 20 Ames St. \\ Cambridge, MA 01239 \\ 1-617-253-8988 \\ joep@media.mit.edu
}

\begin{abstract}
We describe the design of a system of compact, wireless sensor modules meant to capture expressive motion when worn at the wrists and ankles of a dancer. The sensors form a high-speed RF network geared toward real-time data acquisition from multiple devices simultaneously, enabling a small dance ensemble to become a collective interface for music control. Each sensor node includes a 6-axis inertial measurement unit (IMU) comprised of three orthogonal gyroscopes and accelerometers in order to capture local dynamics, as well as a capacitive sensor to measure close range node-to-node proximity. The nodes may also be augmented with other digital or analog sensors. This paper describes application goals, presents the prototype hardware design, introduces concepts for feature extraction and interpretation, and discusses early test results.
\end{abstract}

\section{Keywords}

Interactive dance, wearable sensor networks, inertial gesture tracking, collective motion analysis, multi-user interface

\section{INTRODUCTION}

Several wireless interfaces have been developed to capture dance gestures over the last decade or two. Some have been sensor systems built into shoes, such as the 1980's Taptronics, featuring piezoelectric pickups at the toe and heel [1] and Expressive Footwear by our group at the MIT Media Lab [2]. Originally realized in 1997, this system was an early implementation of a dense, multimodal wireless sensor cluster (now becoming common in sensor networks) that measured 16 variables including many degrees of both contact and free-gesture control. Other examples of wearable dance instrumentation typically use bendable sensors that span primary joints such as the elbows and knees. Architectures of this sort have been introduced by DIEM in

Permission to make digital or hard copies of all or part of this work for personal or classroom use is granted without fee provided that copies are not made or distributed for profit or commercial advantage and that copies bear this notice and the full citation on the first page. To copy otherwise, or republish, to post on servers or to redistribute to lists, requires prior specific permission and/or a fee.

NIME 06, June 4-8, 2006, Paris, France.

Copyright remains with the author(s).
Aarhus [3] and by Mark Coniglio of Troika Ranch in New York [4]. Although these systems have become wireless, they employ a single radio in a beltpack or backpack, hence the various sensors need to be tethered across the body to this central dispatcher. Extreme versions of these types of wearable joint-bend interfaces can be found in full-body motion capture outfits for computer graphics, and flexible fiber-optic angle-sensing systems such as ShapeWrap by Measurand [5].

The systems above were developed for single subjects, and many do not scale well to ensemble performances. For instance, the bandwidth of the Expressive Footwear system was limited $60 \mathrm{~Hz}$ full-state updates for two shoes. Furthermore, no provision was included to sense upper body or arm motion. Some of the centralized backpack systems enable more than one dancer to be accommodated, but the wires running from various sensor locations to the central body-worn transmitter are cumbersome.

Another approach to gesture tracking for dancers avoids any body-worn hardware by exploiting computer vision, processing video from a camera or cameras watching the stage. This technique is now well established, and platforms like the Very Nervous System [6], Eyes Web [7], Big Eye, and Jitter are used by many composers. The prevalence of optical tracking methods has even prompted some artists to develop their own video analysis tools, e.g., [8,9]. This approach is processor intensive, and although the underlying technology and algorithms are steadily improving, computer vision is further limited by constraints on lighting and choreography; robustness to occlusion and background noise remains problematic. Hence, obtaining multiple relevant features reliably from a dance ensemble in a performance setting can be difficult.

Accordingly, we have developed a system of compact wireless inertial sensors that can be worn on the hands and feet of a group of dancers to enable real-time gesture tracking over the entire ensemble. This approach has advantages over other techniques in that each point of measurement has a dedicated wireless connection, the system easily scales to a flexible number of performers and number of points of measurement on the body, does not suffer from occlusion, and provides sensor data which is immediately relevant to features of human motion.

\section{GOALS}

The motivation for this project is the recent opportunity to leverage low-power, high-bandwidth RF solutions and 
compact inertial sensors to create a wearable wireless motion sensing system meeting the demands of many points of measurement and high data rates. Our goal is to implement such a system for an interactive dance ensemble, which is in some ways an ideal situation for pushing high performance requirements. A highly active environment of human motion demands an unrestricting yet sturdy wearable design. Obtaining detailed information about the movement of the human body and the interaction of multiple human bodies demands many points of measurement. Most importantly, using this information as a vehicle for interactive performance, specifically with musical feedback, demands rapid data collection and analysis to achieve a response with a sufficiently low latency. In the broader scope, we hope to test the applicability of this system to other applications, such as analyzing the dynamics of team sports, physical therapy, biomotion measurement and analysis, or personal physical training.

\section{HARDWARE DESIGN}

The current hardware design has its roots in the Stack [10], a modular system, including full IMU card, developed by our research group several years ago as a compact and customizable alternative to our earlier Expressive Footwear design. However, the data radio used at the time was limited to only $115 \mathrm{kbps}$, far too low for our application. Assuming we would like to outfit an ensemble of five dancers wearing sensors on wrists and ankles, with full state updates at $100 \mathrm{~Hz}$, the inertial sensors alone generate:

6sensors $\times 12$ bits/sensor $\times 20$ nodes $\times 100 \mathrm{~Hz}=144 \mathrm{kbps}$.

If we wish to transmit additional information from the capacitive sensors, and account for the increased overhead costs associated with sending small frequent packets for lowlatency, five dancers could easily require up to $400 \mathrm{kbps}$ in practice.

Although compact sensor clusters have been developed at other institutes, none have the characteristics that we need in terms of combining low power and small size with such high data rates. Motes are quite established for sensor networks, but most support mainly peer-peer routing at lower data rates than needed here. Likewise, the Smart-Its and its descendants [11] are designed to work at data rates similar to the Stack. Flety and collaborators at IRCAM [12] have built wireless sensor networks that use a similar transceiver as used in the Stack (and hence also exhibit limited data rate) and others that use the WiFi 802.11 standard, which tends to be much too power hungry for efficient continuous operation with a modest battery. Emmanuel Tapia of the MIT Media Lab has designed very compact wireless accelerometer sensors capable of higher data rates [13], but our application requires more sensor degrees of freedom.

The design presented here includes a full six axis IMU, nodeto-node capacitive proximity sensing, and flexible expansion capabilities, combined with a low power $1 \mathrm{Mbps}$ radio. The sensor node (Fig. 1) measures $4 \mathrm{~cm} \times 4 \mathrm{~cm} \times 2 \mathrm{~cm}$, not including the protruding antenna and external battery pack. As shown, with the battery included, the weight is approximately $45 \mathrm{~g}$. We chose to decouple the battery from the main circuit board, so that it could be affixed to the strap rather than adding to the bulk of the sensor package. This makes the node more comfortable to wear, provides easy access to the battery, and allows for flexibility in the choice of battery pack.

The nRF2401A data radio we utilize is a small, low power, $2.4 \mathrm{GHz}$ device providing up to $1 \mathrm{Mbps}$ data rates. Our communications protocol is a TDMA scheme [14] in which a basestation polls the network for data at the sampling rate, and each node responds within a preprogrammed time slot. The basestation then transmits the data to a central computer via USB for processing. Using this scheme, one basestation can handle full state updates at $100 \mathrm{~Hz}$ for over 25 nodes. This is a significant performance improvement over previous designs. The workable RF range on these devices appears to be on the order of 50 feet, depending on the local RF environment.

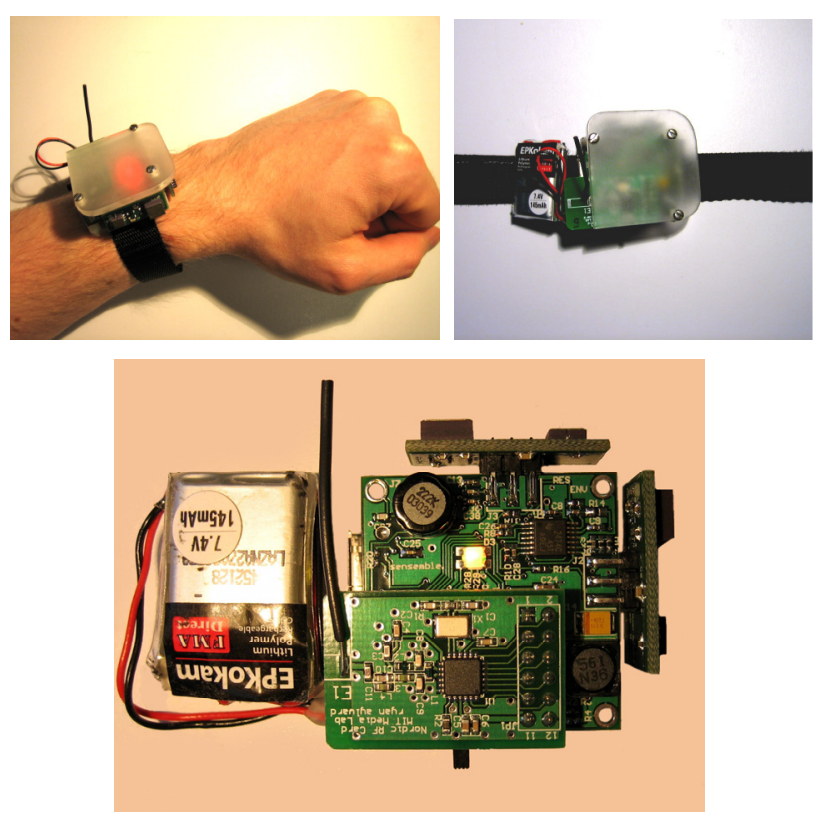

Figure 1. Sensor node on wrist (upper left), removed (upper right), and exposed circuit board (bottom).

The IMU is made up of Analog Devices ADXRS300 rate gyros and ADXL203 accelerometers, as well as associated analog circuitry. Sensor signals are collected by the 12-bit analog to digital converter built into the onboard processor, a TI MSP430F14x. This microcontroller was favored because of its low power consumption, capable A/D, and ample I/O, as well as its use in several of our group's ongoing projects.

The node-to-node capacitive proximity sensor operates by alternating transmit and receive modes on each of the sensor nodes, with only one node transmitting at a time, while the body is grounded. Because of timing constraints, it is not feasible to record measurements for every pair of nodes; rather, several simultaneous transmit nodes and several concurrent receive nodes can be selected in software. During transmit mode, the microcontroller drives an LC oscillator, which generates a high amplitude pulse (tens of volts peakto-peak) at $91 \mathrm{kHz}$. During receive mode, the pulse is picked up by the receiving node, amplified, and sampled in quadrature to estimate its amplitude without the need for phase coherence. The nodes are able to use the same electrode for both transmit and receive modes, thanks to an efficient amplifier circuit inspired by the School of Fish, an electric 
field sensing tool designed several years ago by a former Media Lab student [15]. Capacitive sensing requires an electrode with sizeable area - this could possibly be integrated into the strap securing the sensor package to the body using highly conductive textiles such as Bekiweave [16].

Additional capabilities include a free digital input for interfacing with a Polar heart rate monitor, a free SPI interface for connecting with other digital devices, and a free analog input with associated signal conditioning circuitry for handling an additional resistive sensor, such as a pressure sensor, bend sensor, or light sensor. All of these optional signal lines are broken out to a compact expansion port, which also acts as the programming interface.

Power consumption is always of prime importance in the design of wireless devices; the power source tends to be the largest and most cumbersome component of the system. Unfortunately, our desire to operate continuously with three rate gyros prevents this design from meeting traditional lowpower requirements. Each gyro may consume up to $30 \mathrm{~mW}$, and their slow setup time prevents them from being power cycled. The data radio is also comparatively power hungry, consuming up to $60 \mathrm{~mW}$ in receive mode and $40 \mathrm{~mW}$ in transmit mode, but this can be managed in code by minimizing the amount of time spent in active modes. Ultimately, we chose to operate the system with lithium polymer batteries because they are lightweight, compact, and rechargeable. With two compact $145 \mathrm{mAh}$ cells in series, as pictured above (Fig. 1), the node can operate for four hours on one charge.

\section{RESULTS}

The major advantage of having enough bandwidth to operate multiple sense points on multiple wearers simultaneously is the ability to obtain detailed information about correlated activity within a group. In the context of a dance ensemble, time and spatial correlations can be used to determine which dancers are moving together, which groups are leading or lagging, or perhaps which dancers are responding to one another with complementary movements. With this in mind, our preliminary analysis focuses mainly on the feasibility of extracting simple features that can be used to describe general group dynamics.

\subsection{Correlated Motion}

Previous work has shown that cross-covariance can be used to express both time separation and spatial similarity of gestures performed by multiple users [17]. For example, Figure 2 illustrates pitch gyro data for the hands of three subjects performing a similar gesture in sequence. The locations of the peaks in the associated cross-covariance curves (calculated with respect to subject 1) give the time lags between the three events. In addition, the height of a peak gives a measure of how well the signal shapes are correlated. In this way, we can also obtain a sense for the spatial similarity of the events. Here, subject two does a slightly better job at mimicking the motion of subject one.

One problem with cross-covariance as a feature is that it requires a complete segment of data to calculate. In a streaming situation, windowed cross-covariance must be used, where the window size is chosen to make a tradeoff between latency and the maximum time separation that can be expressed. A feasible use of cross-covariance requiring a short window might be to follow how closely dancers synchronize to music or to a leader, where the delays between their correlated motions are expected to be within a second.

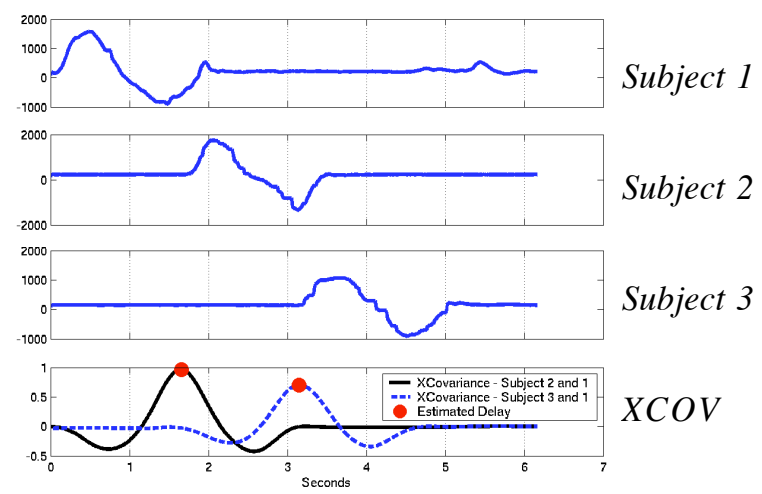

Figure 2. Raw data for hands raised and lowered in sequence (only the pitch gyro is shown) and resulting average cross-covariance.

To test this idea, six sensors were given to three dancers participating in a ballet lesson; each wore one on the right wrist and one on the right ankle. The class then performed an exercise involving a repeated sequence of leg swings executed in unison, to music. Although they were roughly in time with the music, the dancers were not necessarily looking at each other or at an instructor, creating a small but clearly visible delay in their motions (the rehearsal was documented on video for reference). Figure 3 shows a portion of the raw data collected from the leg of each dancer. Because there was very little arm motion associated with this exercise, only leg motion is discussed here. The area from about 35 to 65 seconds corresponds to the synchronized sequence of swings made with the right leg.

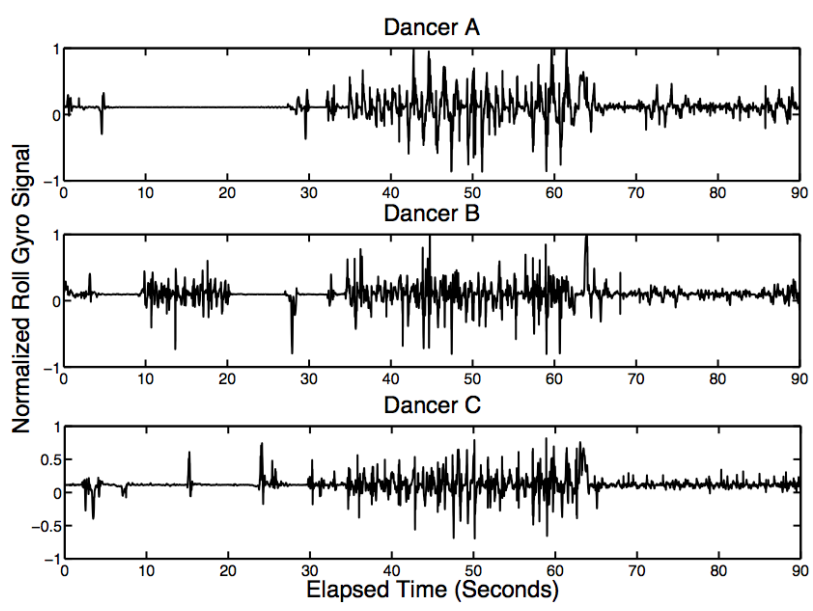

Figure 3. Selected raw data from the ankles of three ballet students performing a sequence of leg swings in unison.

Figure 4 shows the result of windowed cross-covariance analysis on this data segment with a window size of 1 second and a step size of 0.25 seconds. That is to say, at each interval of 0.25 seconds, a window of data was considered, the cross-covariance vector was computed individually for each sensor value, and then the individual vectors were averaged to produce a result. Note that the area of peak crosscovariance, shown in white, tends to waver around the 
baseline as time progresses. This is consistent with the dancers slowly leading and lagging with respect to one another by small amounts. Because the step size is small enough, individual leg swings and their synchronicity across the ensemble can be picked out. It is clear from the relatively stable middle plot that Dancer A and Dancer C were closely synchronized for the duration of the exercise, while Dancer B fluctuated from about 0.3 seconds ahead of Dancer A to 0.3 seconds behind Dancer A. This fluctuation reflects accurately what is visible in the video. Interestingly, it turns out that Dancers $\mathrm{A}$ and $\mathrm{C}$ were facing each other during the exercise, while Dancer B had her back turned to the others.

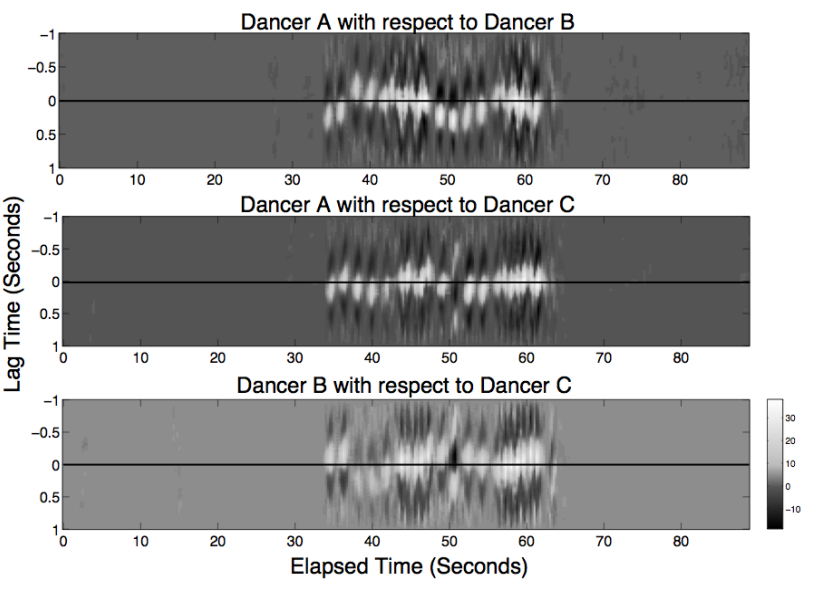

Figure 4. Windowed cross-covariance (averaged across sensor values) between pairs of dancers, for the data segment presented in Figure 3.

\subsection{Quantifying Activity}

In addition to extracting correlations between the activities of a group, it is important to obtain information about the properties of the activities being observed. These properties might include variations in the overall activity level of an individual or group at different time scales, principal axes of movement, or other features extracted during an interval of high activity.

One approach to activity measurement involves computing the average running variance for various combinations of sensors on individual nodes. If the separation between gestures is long enough, variance spikes can be used to delineate them. In other cases it might be useful to use a lowpass filter to obtain an envelope on the running variance, in order to determine slower trends in the activity level. For example, data was collected from the right wrist and ankle of a ballet student performing a sequence of motions in which slow kicks with the right foot transitioned into fast, tense kicks (in ballet terminology, petit battement). The full sequence is framed with a stylistic tension and release of the right arm at the beginning and end, respectively. Figure 5 shows a portion of the raw data from this segment along with four different activity envelopes obtained from the windowed variance of both upper and lower body movement. Accelerometer activity here denotes the average variance across the accelerometer axes, while rotational activity denotes the average across the gyro axes. One can clearly see a marked increase in activity as leg motion transitions to faster kicking. The role of the arm movement is apparent in the activity envelope as well.
Similar conclusions can be drawn from figure 6, which illustrates the activity envelopes of leg motion for each dancer during the period of correlated activity highlighted earlier in figures 3 and 4 . Two areas of peak activity across the ensemble appear around 50 and 60 seconds into the sample, corresponding to repeated leg swings over the full range of motion from front to back and back to front. The general trend of activity is increasing over the segment from 30 seconds to 60 seconds, as the instructor urges the dancers to make each leg swing "successively higher". Finally, we see activity for Dancer B in the interval from 10 to 20 seconds that is not reflected in the movements of the other dancers, corresponding to a few "warm-up" leg swings by Dancer B. Comparison of the activity levels is all that is required to flag this unique period of activity, at which point it could be analyzed more closely, or used as evidence that Dancer B should be clustered in a different subgroup from Dancers A and C. Note that the cross-covariance analysis shown in figure 4 is unable to compare the warm-up leg swings with motion occurring later in time, because the window is only 1 second long. Given enough storage and computing power, one solution is to save interesting data segments for correlation with future data, or to monitor running cross-covariance on multiple time scales.

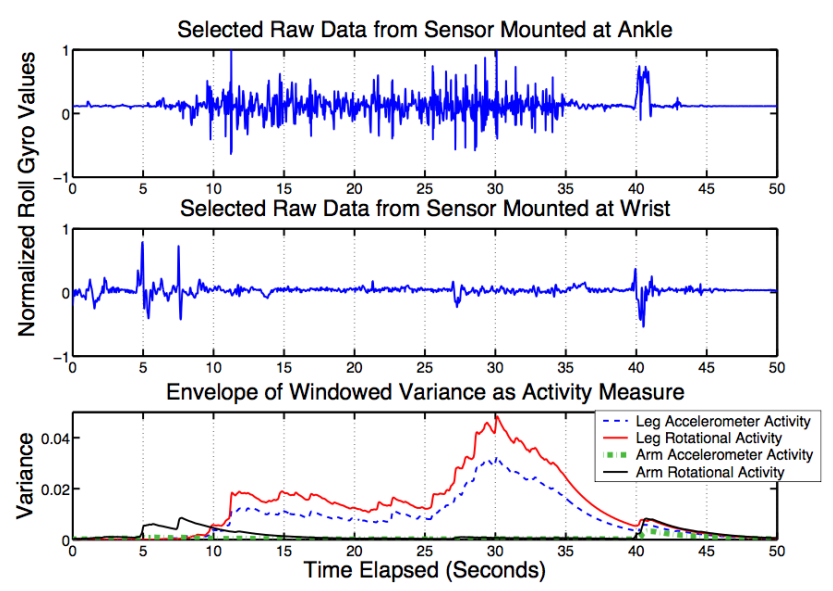

Figure 5. Selected data and resulting activity envelopes as dancer transitions from slow kicks to rapid tense kicks. Sequence of leg motions is framed by stylistic arm motion.



Figure 6. Activity envelopes for the synchronized leg movement highlighted in Figures 3 and 4. 
Looking at figure 5 and 6 , it would seem as if there is no reason to distinguish between accelerometer and gyro activity. Indeed, sensor activity on a single node is often highly correlated, because human motion is unlikely to occur along only one axis. The accelerometers are also subject to gravity and centripetal acceleration, so rotations will be picked up strongly in some cases. It should be possible to use the gyro signals to help isolate translational acceleration from other types of movement picked up by the accelerometers. However, if one wishes to identify specific classes of activity, it may be more important to compare motion along each axis than rotational versus translational motion. One approach is to keep track of which sensor has the highest variance on each node or on each individual, with the goal of analyzing activity one person at a time. A more efficient approach might be to create a group feature such as mean activity on each sensor axis, for each limb, across the entire ensemble, to determine the predominate axes of collective motion.

For example, figure 7 demonstrates the results of a group of three people raising and lowering their right hands in unison. The bottommost plot indicates the variance on each sensor axis for the right arm, averaged across all three subjects. Note that the average variance of the pitch gyro dominates. This supports our intuition that the act of raising and lowering the hand involves mostly a rotation in pitch. Extracting this information from average windowed variance may simplify the task of detecting specific gestures by determining which sensor signals are most important, or by defining a subgroup that is performing a similar gesture before applying heavier analytical techniques. One can also imagine a situation in which the correlation measurements discussed above are desired, but it is unclear who should be interpreted reasonably as a "reference" for the rest of the group. By comparing the average group variance to the individual variance, one can determine if the motions of a specific subject are characteristic of the entire group, or lie outside the norm.



Figure 7. Right arm pitch gyro signals and windowed variance averaged across subjects for each sensor axis, as hands are raised and lowered in unison.

\subsection{Capacitive Sensor}

One of the limitations of small-scale inertial sensing is that it is extremely difficult to obtain a reference frame for any sort of position tracking. Yet, the shape of the body may be a more intuitive communication tool than the dynamics of the body. To supplement inertial data with information even as simple as "arms together" and "arms apart" would add significant depth to the interface. This was the idea behind the node-to-node capacitive proximity sensor.

Initial performance evaluations have determined that the capacitive system suffers from a very nonlinear response, which, coupled with high noise levels, limits its useful range (Fig. 8). Despite this, nodes grounded through the user's body can be sensed up to a spacing of $30 \mathrm{~cm}$ with a $16 \mathrm{~cm}^{2}$ electrode. Nodes that do not share a ground, i.e. worn by different individuals, have reduced range but can still be detected. Past attempts at similar sensing systems have achieved better range, possibly due to higher voltage output on the transmitting electrode $[15,18]$. It may thus be possible to improve the performance with minor adjustments.



Figure 8. Typical response of the capacitive sensing system.

In the current version, because of the reduced sensitivity beyond about $5 \mathrm{~cm}$, it may not be efficient to transmit a full 12-bit value for every capacitive measurement. Rather, the signal should be compressed to fit a range of 8 or fewer bits with a more linear response. Another possibility is to use the existing response to form a simple one-bit indication of close versus distant. Until improvements can be made, this scheme fulfills the minimum requirements. In either case, data reduction will enable the transmission of capacitive measurements from more nodes without compromising the bandwidth available for higher priority sensor data.

\section{GENERATING MUSICAL FEEDBACK}

To demonstrate the utility of the system as a multi-user interface for interactive performance, it will be necessary to map extracted activity features to musical sound in a satisfying way. In a traditional free gesture interface, each degree of freedom might be mapped directly to a specific continuous control or set of event triggers. In this system, however, there are at least six degrees of freedom per node provided by the inertial sensors, and typically four nodes per user, making direct mapping impractical. Taking the first step towards a practical strategy for musical mapping in this framework, we have been focusing on forming descriptions of motion at the group level rather than at the individual level. As suggested above, simple group features can express 
a whole range of useful information, such as who is leading and who is following, degree of correlation across the ensemble, changes in activity level across the ensemble, the existence of subgroups or clusters within the ensemble that could be considered separately, principal axes of activity within subgroups, the location of an event unique to one individual, or relationships between levels of upper body motion and lower body motion. In turn, the treatment of the ensemble as an organic unit offers new possibilities for musical interpretation.

However, the potential amount of information expressed by these group features alone is still too large for a direct mapping to music. The problem can be simplified by interpreting group dynamics in the context of a specific piece. For example, the music can be generated from a loose framework or score designed alongside the choreography. At a given point in the score, one may be looking for a specific set of possible changes in the dancers' movements that signal musical events such as changing timbral qualities, the entrance of a new melodic line, or a shift to a new section. By placing contextual limits on the decision space, pattern recognition algorithms can be trained on a specific performance to streamline the control process. Although the dancers do not actually generate music directly under this model, they are able to freely control their progression through sections of the score, alter their interpretation of the context, and add embellishments. This approach should provide a balance between musical continuity and the sense of causality between the movements of the dancers and the generated sound, which is essential for an engaging interactive performance.

One limitation to address is the fact that many of the features discussed in this paper are slowly varying, or have a significant amount of latency associated with them. This is unsuitable for triggering sudden events or percussive sounds, as the human tolerance to latency in this case is quite low. It may be possible to train a state-based gesture-tracking model that would allow for rapid activity detection by predicting future states, but applying a simple threshold on one or more continuous features may be a better option.

\section{CONCLUSIONS \& FUTURE WORK}

In this paper, we have presented a compact, wearable sensor system enabling real time collective activity tracking for interactive dance. The sensor node comprises a full 6 -axis inertial measurement unit with supplementary capacitive node-to-node proximity sensing. Preliminary results demonstrate that our design is viable for analyzing a wide range of collective activity parameters in a dance setting. As the current $\pm 1.7 \mathrm{~g}$ accelerometer was found to have insufficient range to capture certain quick motions, it will be replaced by the $\pm 10 \mathrm{~g}$ ADXL210E. We also hope to increase the range of the capacitive sensor. Future work will focus on adding to the feature set developed here, assessing real-time operation with special attention to low-latency requirements, and developing a more specific framework for music generation with implementations in Max/MSP or PD.

\section{REFERENCES}

[1] di Perna, A. Tapping into MIDI. Keyboard Magazine (July 1988), p. 27.

[2] Paradiso, J., et al. Design and Implementation of Expressive Footwear. IBM Systems Journal, 39(3\&4) (October 2000), pp. 511-529.

[3] Siegel, W. and Jacobsen, J. The Challenges of Interactive Dance: An Overview and Case Study. Computer Music Journal 22, No. 4 (1998), 29-43.

[4] Coniglio, M. The MidiDancer system, see: http://www.troikaranch.org/mididancer.html

[5] See: http://www.measurand.com

[6] Zacks, R. Dances with Machines. Technology Review (May/June 1999), pp. 58-62.

[7] Camurri, A., et al. EyesWeb - Towards Gesture and Affect Recognition in Dance/Music Interactive Systems. Computer Music Journal, 24(1), pp. 57-69.

[8] Downie, M. Choreographing the Extended Agent: Performance Graphics for Dance Theater. Ph.D. Thesis, MIT Media Lab, September 2005.

[9] Wechlser, R., Weiss, F., and Dowling, P. EyeCon - A motion sensing tool for creating interactive dance, music, and video projections. In Proc. of the SSAISB Convention, (Leeds England, Mar. 29, 2004).

[10] Benbasat A.Y. and Paradiso, J.A. A Compact Modular Wireless Sensor Platform. In Proc. of the 2005 Symposium on Information Processing in Sensor Networks (IPSN), (Los Angeles, CA, April 25-27, 2005), pp. 410-415.

[11] Holmquist, L.E., et al. Building intelligent environments with Smart-Its. Computer Graphics and Applications, IEEE (Jan/Feb 2004), pp. 56-64.

[12] Flety, E. The WiSe Box: a Multi-performer Wireless Sensor Interface using WiFi and OSC. In Proc. of NIME 05, (Vancouver Canada, May 26-28, 2005), pp. 266-267.

[13] Munguia Tapia, E., et al. MITes: Wireless portable sensors for studying behavior. In Proceedings of Extended Abstracts Ubicomp 2004: Ubiquitous Computing (2004).

[14] Lovell, S.D. A System for Real-Time Gesture Recognition and Classification of Coordinated Motion. M. Eng. Thesis, MIT EECS Dept., Jan. 2005.

[15] Smith, J.R. Electric Field Imaging. Ph.D. Thesis, MIT Media Lab, February 1999.

[16] See: http://www.bekaert.com/bft

[17] Aylward, R., Lovell, S., Paradiso, J. A Compact, Wireless, Wearable Sensor Network for Interactive Dance Ensembles. Submitted to Int. Workshop on Wearable and Implantable Body Sensor Networks, November 2005.

[18] Paradiso, J., Gershenfeld, N. Musical Applications of Electric Field Sensing. Computer Music Journal, 21(2), (Summer 1997), pp. 69-89. 